\section{Novel anti-tuberculosis drug designs by data mining for similarity in substituent substitution and structure modification}

\section{Ronald Bartzatt}

Chemistry Department, Durham Science Center, University of Nebraska, Omaha, NE, USA

\section{Abstract}

Mycobacterium tuberculosis (TB) is among the most common of infectious diseases that cause death, and as many as one-third of the world's population may be infected. This work presents 17 novel hydrazide agents formed by focused in silico data mining utilizing search parameters restricted to substituent replacement only. Substituent substitution has been highly successful in design of novel antibacterial and antiviral drugs. This diverse set of hydrazide constructs possess molecular properties indicating favorable bioavailability with excellent intestinal absorption for oral administration. All agents have zero violations of the Rule of 5 , indicating favorable druglikeness. Important pharmaceutical properties including polar surface area, Log $\mathrm{P}$, and formula weight were determined and compared to that of the parent structure of isoniazid by hierarchical cluster analysis and discriminant analysis. The average Log $\mathrm{P}$ with range is -0.258 and -2.165 to 1.373 , respectively. The average polar surface area (PSA) with range is $75.19 \mathrm{~A}^{2}$ and $55.121 \mathrm{~A}^{2}$ to $94.036 \mathrm{~A}^{2}$, respectively. The diverse range of PSA and Log P, with other descriptors, portend a versatile group of hydrazide drugs having substantial potential to expand the application and effectiveness forclinical treatment of multi-organ infected TB patients. Analysis of similarity indicated that all 17 agents are significantly similar to isoniazid, however discriminant analysis and hierarchical cluster analysis are able to differentiate isoniazid based upon molecular properties. Molecular weight and number of atoms were highly correlated by Pearson $r(r>0.9000)$, with Log P moderately correlated $(r>0.5500)$ to number of atoms, molecular weight, and volume. Seventeen hydrazide compounds (success rate of approximately 38\%) having diverse pharmaceutical properties resulted from substituent data mining with potential for clinical application.

\section{Introduction}

Mycobacterium tuberculosis (TB, tubercle bacillus) is a type of mycobacteria that causes a common and dangerous infectious disease in humans, which usually attacks the lungs. ${ }^{1,2}$ Saliva of infected individuals transmitted by cough, sneeze, sputum, or other methods spreads the infectious bacteria. ${ }^{2}$ Although generally associated with a pulmonary locality, the bacillus can infect other organs of the body and cause a broad range of symptoms. It is generally believed that up to one-third of the world's population is infected with TB. ${ }^{3}$ Infections of other organs can co-exist with a pulmonary located TB, however $75 \%$ of all cases involve infection of the lungs. ${ }^{4}$ The high lipid content within the cell wall of this microbe contributes to the unique clinical features of this pathogen. ${ }^{5}$ In itself Mycobacterium tuberculosis is a small aerobic non-motile bacillus classified as a gram-positive bacterium, and is able to survive in dry states for weeks (which can exacerbate the infectious propensity), and is able to withstand weak disinfectants. Other TB causing mycobacterium that comprise the Mycobacterium tuberculosis complex include: M. africanum (a significant problem in some parts of Africa), M. canetti (which is observed in Africa), M. microti (observed in immunodeficient patients), and M. bovis (which has been reduced through pasteurization of milk). ${ }^{6}$ TB is among the top three infectious killer diseases (malaria, HIV, TB) with several million new cases of TB take place every year. ${ }^{7}$ The global resurgence of TB and rapid emergence of multi-drug resistant TB (MDR-TB) accentuates the need for development of new antituberculosis compounds. The investigation of new pharmaceutics to content with MDR-TB is urgently necessitated. ${ }^{7}$ Previous studies have shown that $1.6 \%$ of global new cases of TB are MDR-TB, and in 2006 as much as 50\% of new cases occurring in China and India. ${ }^{8}$ If cure rates of MDR-TB do not increase than the appearance of extensively drug resistant (XDR-TB) cases will increase greatly. ${ }^{8}$ Studies of drug resistance increases the efficiency of detection and can generate new targets for drug development. ${ }^{9}$ MDR-TB is recognized as an emerging problem in Nigeria in which $8 \%$ of diagnosed cases were MDR-TB, and it is strongly associated with previous treatment. ${ }^{10}$ Kazakhstan is one of 14 nations having high morbidity to MDR-TB with resistance to isoniazid and rifampicin. ${ }^{11}$ In 2006 the World Health Organization estimated that nearly 500,000 cases of MDR-TB occurred with new cases of XDR-TB (a MDR-TB instance that is also resistant to a fluoroquinolone and at least one second line agent) reported in 45 nations on all five continents. ${ }^{12}$ High rates of XDR-TB was reportedly observed in India. ${ }^{13}$ From the time period of 1993 to 2007 there were a reported 83 cases of XDR-TB. ${ }^{14}$ Cases of XDR-TB have poor outcomes in treatment, prolong periods of
Correspondence: Dr. Ronald Bartzatt, Chemistry Department, Durham Science Center, University of Nebraska, 6001 Dodge Street, Omaha Nebraska 68182 USA.

Tel: +1.402.554-3612 - Fax: +1.402.554-3888.

E-mail: rbartzatt@unomaha.edu

Key words: tuberculosis, tuberculostatic, isoniazid, hydrazide.

Acknowledgements: this study was supported by the College of Arts and Sciences, University of Nebraska, Durham Science Center and Chemistry Department.

Received for publication: 18 July 2011. Revision received: 7 October 2011.

Accepted for publication: 13 October 2011.

This work is licensed under a Creative Commons Attribution NonCommercial 3.0 License (CC BYNC 3.0)

CC Copyright R. Bartzatt, 2011

Licensee PAGEPress, Italy

Drugs and Therapy Studies 2011; 1:e15 doi:10.4081/dts.2011.e15

infection, and more limited treatment options, conditions that call for new pharmaceutics and clinical treatment options. ${ }^{15}$ Investigations have concluded that the emergence of XDR-TB is also a reflection of poor TB management, making the current state of affairs very alarming. ${ }^{16}$ Tuberculosis occurring in the spine is a leading spine pathology in India, which causes paraplegia and progressive deformity. ${ }^{17}$ Tuberculosis infections of the spine is the most common extrapulmonary form in the Asian subcontinent. ${ }^{18}$ Clearly novel drug designs are advised and urgently necessary in order to confront the onslaught of tuberculosis, inclusive of the MDR-TB and XDR-TB cases. The modification of the fundamental isoniazid scaffold has been shown previously to generate potent and usable anti-tuberculosis agents. ${ }^{19}$ The modification of known, effective, and established drugs can be an effective approach for rational drug design. ${ }^{19}$ This study presents an approach for structure directed drug design to produce hydrazide agents that will have beneficial molecular properties for the clinical treatment of tuberculosis.

\section{Materials and Methods}

\section{Molecular modeling and assembly of constructs}

Molecular modeling (2-dimensional) for structure visualization and analysis of substituent steric characteristics (i.e. Validation 
of substituent size and position) was accomplished utilizing ACD/ChemSketch modeling v. 10.00 (Advanced Chemistry Development, 110 Yonge Street, Toronto Ontario, M5C 1T4 Canada). The actual computer aided in silico structure search for the substituent utilized for placement within the parent scaffolding is accomplished by pursuing a chemical substructure and similarity search available through Molinspiration Cheminformatics by Java (Molinspiration Chemiformatics, Nova ulica 61, SK-900 26 Slovensky Grob, Slovak Republic). Various molecular properties such as polar surface area, violations of Rule of 5 , molecular volume, number of oxygens and nitrogens and amines and hydroxyls, necessary for evaluating drug likeness of hydrazide derivates can be determined by Molinspiration Properties Calculations module. In addition, these various molecular properties can be ascertained by use of the in silico approach by MolSoft L.L.C. (Molsoft 11199 Sorrento Valley Road, S209 San Diego CA 92121).

\section{Pattern recognition and elucidation}

To identify underlying associations and patterns within the molecular descriptors, such a multivariate numerical data matrix is examined by appropriate pattern recognition techniques. Included in this analytical operation is hierarchical cluster analysis, which was accomplished by and available through KyPlot v. 2.0 Beta 15 (copyright Koichi Yoshioka 1997 2001). Other efficacious pattern recognition methods for elucidation of numerical relationships include discriminant analysis and
ANOSIM (analysis of similarity), both of which were performed and available by PAST v. 1.80 (copyright Oyvind Hammer, D.A.T. Harper 1999-2008). PATH analysis was applied for determining cause and effect relationships is effectively accomplished by the online available OpenStat (copyright Nov. 20 2010, William G. Miller).

\section{Numerical analysis of multivariate data matrix}

Statistical analysis and Pearson $r$ determination of all numerical data was performed by Microsoft EXCEL (EXCEL 2003, copyright 19852003). Screening for numerical outliers was done by Grubb's Test (extreme studentized deviate) by online available GraphPad Software (2236 Avenida de la Playa, La Jolla, CA 92037 USA). The necessary multiple regression analysis was performed by GraphPad InStat v. 3.0 software for Windows 95 (HJ Motulsky, GraphPad InStat 3.0 GraphPad Software, Inc., San Diego California USA, www.graphpad.com)

\section{Results}

Implementing a data mining operation for search of similar substituents (see Materials and Methods) ultimately resulted in 17 constructs presented in Figure 1 (as agents 2 through 18). Note that agents 1 through 18 have retained the hydrazide functional group $\left(-\mathrm{C}(0) \mathrm{NHNH}_{2}\right)$ and that this functional group

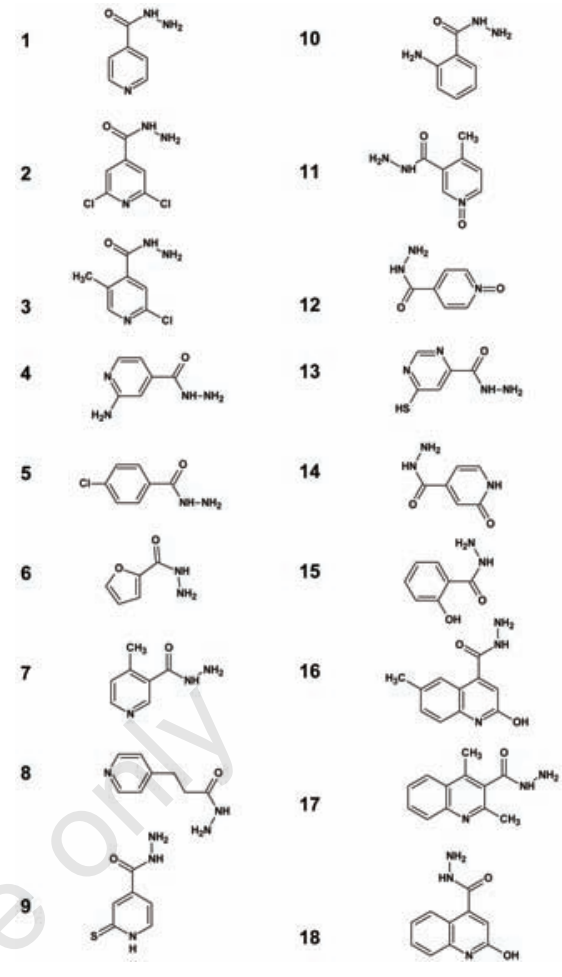

Figure 1. Molecular scaffolding of isoniazid (1) and 17 analogs determined by in silico similarity and substituent search and optimization. All compounds have a single hydrazide group $\left(-\mathrm{C}(\mathrm{O}) \mathrm{NHNH}_{2}\right)$. Other functional groups include hydroxyl groups $(-\mathrm{OH})$, amines $\left(-\mathrm{NH}_{\mathrm{n}}\right)$, and furan. The genre of substitution affects water solubility, $\log P$, and polar surface area.

Table 1. Molecular properties of tuberculostatic agents.

\begin{tabular}{|c|c|c|c|c|c|c|c|c|c|}
\hline Agents & $\begin{array}{c}\log P \\
A^{2}\end{array}$ & $\begin{array}{c}\text { Polar } \\
\text { Surface } \\
\text { area }\end{array}$ & $\begin{array}{l}\text { Number } \\
\text { of atoms }\end{array}$ & $\begin{array}{l}\text { Molecular } \\
\text { weight }\end{array}$ & $\begin{array}{l}\text { Number of } \\
\text { oxygens \& } \\
\text { nitrogens }\end{array}$ & $\begin{array}{l}\text { Number of } \\
\text { Hydroxyls } \\
\text { \& Amines }\end{array}$ & $\begin{array}{l}\text { Number of } \\
\text { Violations } \\
\text { Rule of } 5\end{array}$ & $\begin{array}{c}\text { Number } \\
\text { Rotatable } \\
\text { Bonds }\end{array}$ & $\begin{array}{c}\text { Volume } \\
\left(\mathrm{A}^{3}\right)\end{array}$ \\
\hline 1 Isoniazid & -0.969 & 68.013 & 10 & 137.14 & 4 & 3 & 0 & 1 & 122.56 \\
\hline 2 & 1.071 & 65.013 & 12 & 206.03 & 4 & 3 & 0 & 1 & 149.63 \\
\hline 3 & 0.48 & 68.013 & 12 & 185.61 & 4 & 3 & 0 & 1 & 152.66 \\
\hline 4 & -1.144 & 94.036 & 11 & 152.16 & 5 & 5 & 0 & 1 & 133.85 \\
\hline 5 & 0.998 & 55.121 & 11 & 170.60 & 3 & 3 & 0 & 1 & 140.25 \\
\hline 6 & -0.422 & 65.261 & 9 & 126.12 & 4 & 3 & 0 & 1 & 108.29 \\
\hline 7 & -0.54 & 68.013 & 11 & 151.17 & 4 & 3 & 0 & 1 & 139.12 \\
\hline 8 & -0.997 & 68.013 & 12 & 165.20 & 4 & 3 & 0 & 3 & 156.17 \\
\hline 9 & -0.961 & 70.912 & 11 & 169.21 & 4 & 4 & 0 & 1 & 139.57 \\
\hline 10 & -0.245 & 81.144 & 11 & 151.17 & 4 & 5 & 0 & 1 & 138.01 \\
\hline 11 & -1.788 & 80.58 & 12 & 167.17 & 5 & 3 & 0 & 1 & 147.63 \\
\hline 12 & -2.165 & 80.58 & 11 & 153.14 & 5 & 3 & 0 & 1 & 131.07 \\
\hline 13 & -0.631 & 80.905 & 11 & 170.20 & 5 & 3 & 0 & 1 & 136.07 \\
\hline 14 & -1.303 & 87.983 & 11 & 153.14 & 5 & 4 & 0 & 1 & 130.69 \\
\hline 15 & 0.809 & 75.349 & 11 & 152.153 & 4 & 4 & 0 & 1 & 134.74 \\
\hline 16 & 1.103 & 88.241 & 16 & 217.23 & 5 & 4 & 0 & 1 & 191.13 \\
\hline 17 & 1.373 & 68.013 & 16 & 215.26 & 4 & 3 & 0 & 1 & 199.68 \\
\hline 18 & 0.679 & 88.241 & 15 & 203.20 & 5 & 4 & 0 & 1 & 174.57 \\
\hline
\end{tabular}

$\mathrm{A}^{2}$, Angstroms ${ }^{2} ; \mathrm{A}^{3}$, Angstroms ${ }^{3}$ 
is accompanied with other variations in structure. Agents 2 and 3 gained halogen atom chlorine, whereas 15,16 , and 18 gained hydroxyl groups $(-\mathrm{OH})$. Compounds 11 and 12 contain pyridine 1-oxide moiety. Agents 16,17 , and 18 contain quinoline moiety. These in silico derived compounds analogous to isoniazid do present a diverse set of drug-like compounds nevertheless. For interactive comparison with other agents and to the parent compound various molecular properties are determined and are presented in Table 1. Nine descriptors which are significant indicators of druglikeness and bioavailability are presented in Table 1 for all 18 hydrazide agents. Log $\mathrm{P}$ is moderately correlated $(0.4500<\mathrm{r}<0.7500)$ with number of atoms, formula weight, and volume. Molecular volume is very highly correlated ( $r>0.9000$ ) with number of atoms and formula weight. The numerical range of $\log \mathrm{P}$ is 3.538 (low -2.165, high 1.373), a broad range for this in silico optimization (mean -0.258), but indicating the versatile possibilities of modifications to isoniazid. In addition, the polar surface area varies from low of 55.121 to high 94.036 , a range of 38.915 or $51.76 \%$ of the mean value, confirming the substantial potential for variation of the parent isoniazid structure. The number of oxygens, nitrogens, hydroxyls, and amines comport as hydrogen bond acceptors and donors, enabling aqueous solubility. Utilizing multivariate properties matrix Table 1 then a vertical dendrogram following hierarchical cluster analysis outcome showing relationships of all 18 agents is shown in Figure 2. This outcome using Euclidean distance (linear direct distance between two points) and single linkage conditions (the minimum distance between elements of each cluster). Discriminant function analysis is used to classify with the results shown having two groups whose members are determined to be most analogous from properties (variables) presented in Table 1. The two groups are as follows: i) agent 1 (isoniazid), 3 , $4,7,8,11,12,13,14$, and 17; ii) agent $2,5,6$, $9,10,15,16$, and 18. An additional important relationship that is shown in Figure 3 within formula weight and partition coefficient $\log P$ is a general increase of $\log P$ numerical values with greater formula weight. Substituent data mining incurs an increase of $\log \mathrm{P}$ as formula weight varies from low to high.

\section{Discussion}

Estimated that one-third of the world's population is infected with $\mathrm{TB}^{3}$ with infections of other organs in addition to pulmonary infections emphasizes the requisite for alternate drug moieties having focused physiological application. Expressed otherwise, the state of global infection compels the development of additional drug designs having versatile or commodious pharmacodynamics. The contriving of novel constructs initiating from previous effective drug structures is a common methodology for achieving rational drug design. Isoniazid, or pyridine-4-carbohydrazide, is a first-line anti-tuberculosis medication utilized in prevention and treatment of TB. Isoniazid achieves MIC60 of Mycobacterium tuberculosis at concentrations less than $40 \mu \mathrm{g} / \mathrm{mL}^{19}$ Contriving additional drug scaffolds that are modeled following after isoniazid should produce potentially effective clinical tuberculostats. Previous studies have demonstrated the substantial effectiveness of polar surface area (PSA) for prediction of drug transport mediated by intestinal absorption by modeling established drug-like agents properties. ${ }^{20}$ Specifically the greater the PSA will inhibit intestinal absorption of an orally administered drug. Namely a PSA greater than $110 \mathrm{~A}^{2}(\mathrm{~A}=$ angstroms) suggests an intestinal absorption of less than $20 \%$ of total amount of drug present. ${ }^{20}$ The PSA values vary from $55.121 \mathrm{~A}^{2}$ to $94.036 \mathrm{~A}^{2}$ for expected intestinal absorption that varies from $>85 \%$ to approximately $35 \%$, respectively. To evaluate druglikeness better and extend profiling methods to lead-like properties of compounds, to achieve better starting points in early drug discovery and for saving of time and cost, the Rule of 5 was introduced. ${ }^{21}$ In general an orally active drug has no more than one violation of the following criteria: ${ }^{21} \mathrm{i}$ ) not greater than 5 hydrogen bond donors (i.e. $-\mathrm{OH}$, -NHn); ii) not more than 10 hydrogen bond acceptors (oxygen and nitrogen atoms); iii) a formula weight not greater than 500 ; and 4) Log P not greater than 5 . All 18 agents presented here have zero violations of Rule of 5 , thereby indicating favorable druglikeness and bioavailability. Previous studies of oral absorption and brain penetration related to and as a function of polar molecular surface area have shown brain penetration decreases with increasing polar surface area. ${ }^{22}$ Orally active drugs which are transported passively through the transcellular route should not exceed a PSA of approximately $120 \mathrm{~A}^{2}{ }^{22}$ Polar surface area is a dominating factor for oral absorption and brain penetration of drugs which are transported particularly by the transcellular route. ${ }^{22}$ Drugs having greatest potential for brain penetration will have a polar surface area of 60 to $70 \mathrm{~A}^{2}{ }^{22}$ This criteria is met by agents 1 (isoniazid), 2, 3, 5, 6, 7, 8, and 17; which thereby would be useful in the treatment of Mycobacterium tuberculosis infections of the central nervous system. The purpose of cluster analysis is to discover a system of organizing objects, into groups in which members within any group possess similarity in properties. ${ }^{23}$ Presented in Figure 2 are these drug agents (denoted as Objects) which are clustered (grouped) according to greatest similarity to other agents. Super node A is the initial parent cluster followed by node B having agents 16 , 18 , and 17 (most similar). Super node C contains isoniazid (1) with closest drug 6, 7, and 8 , followed by 2 and 3 . Analysis of similarities (ANOSIM) provides a way to test statistically whether there is a significant difference between two or more groups of sampling units (drugs) ${ }^{24} \mathrm{~A}$ large positive $R$ (up to 1 ) signifies dissimilarity between groups. Using properties shown in Table 1 the ANOSIM result $\mathrm{R}=0.2315$, indicates an appreciable level of similarity among these compounds, a conclusion expected due to the application of isoniazid as the

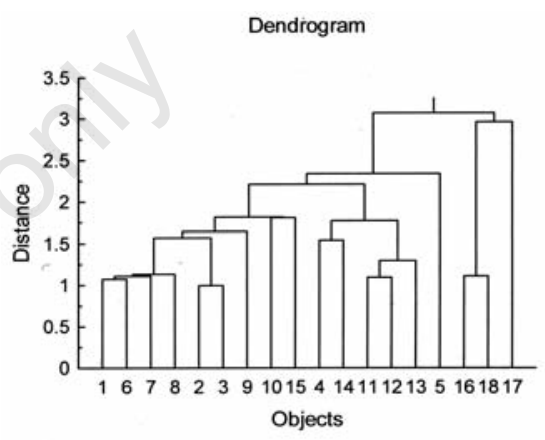

Figure 2. Vertical hierarchical cluster analysis outcome showing relationships of all 18 agents based on molecular properties. Note that Objects (drugs) are clustered (grouped) according to greatest similarity to other constructs. Super node A is the parent cluster followed by node B having agents 16 , 18, and 17 (most similar). Super node C contains isoniazid (1) with closest drug 6,7, and 8 , followed by 2 and 3 .

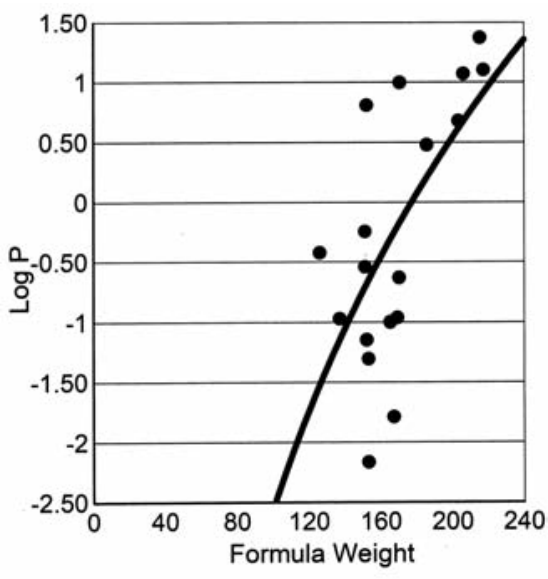

Figure 3. The 2-way plot demonstrating the relationship of formula weight of these constructs compared to $\log P$ is not random. As formula weight increases the numerical value of $\log P$ increases. Equation of line: $y$ $=447 \operatorname{Ln}(x)-2317$, with the correlation of polynomial fit as $r=0.6600$. 
search parameter within the in silico substituent mining. Discriminant function analysis utilizes differentiating variables to classify two or more naturally occurring groups. ${ }^{23}$ The end result of this type of analysis is a model that allows prediction of group membership when only the interval variables are known. ${ }^{23}$ Again the two groups are as follows: i) agent 1 (isoniazid), 3, 4, 7, 8, 11, 12, 13, 14, and 17; ii) agent $2,5,6,9,10,15,16$, and 18. Although somewhat different than the results of hierarchical cluster analysis, the outcome illuminates the compounds most like isoniazid, therefore expected to have similar pharmacodynamics.Note that another relationship exists that is shown in Figure 3, that formula weight and partition coefficient Log $\mathrm{P}$ is generally increasing in Log $\mathrm{P}$ values along with greater formula weight. This line is defined by equation: $\mathrm{y}=447 \operatorname{Ln}(\mathrm{x})-2317$, having a correlation of polynomial fit to be $r=0.66$. Even so the variation in partition coefficient $\log P$ that follows concurrent variation of formula weight brings about modified but efficacious tuberculostatic pharmaceutics varying in routes of administration and permeability in cell membranes which could enhance efficacy of clinica treatment. ${ }^{19,21}$ Potentially the improvement of pharmacokinetics offset the impact of drug resistant Mycobacterium tuberculosis morbidity and mortality. In general the purpose of multiple regression is to learn more about the relationship between several independent or predictor variables and a dependent or criterion variable. Investigators will apply multiple regression when trying to predict some out come or criterion variable. The premise of multiple regression is similar to that of simple linear regression, however, in multiple regression, interest lies in more than one predictor of our criterion variable. Applying the properties found in Table 1 produces a regression equation as follows: formula weight $=-5.133+$ (69.639)(number of oxygen \& nitrogens) + (25.710)(number of $-\mathrm{OH} \&-\mathrm{NH} 2)$ (1.616)(number rotatable bonds) + $(0.8651)$ (volume $)+(8.430)(\log \mathrm{P})-$ (4.517)(polar surface area). The $\mathrm{R}$ squared value of 0.9231 account for $92.31 \%$ of variance of formula weight explained in the model. of the independent (predictor) variables volume, Log P, polar surface area and volume are most significant contributors. Additional analogs to isoniazid may be elucidated by this equation by the descriptors obtained within the in silico data mining of this study. The pursuit of studies for design of novel anti-tuberculosis drugs may include in silico data mining algorithms that focus on substituent substitution while preserving the functional group responsible for bacteria death. The results as established here produce small molecule hydrazide compounds with variation in substituent that are sufficient for efficacious modification of important pharmacological properties determined to be crucial in favorable druglikeness, bioavailability, and intestinal absorption. Isoniazid is a successful first-line anti-tuberculosis drug from which analogous hydrazide entities can be determined and having molecular properties suitable for clinical application, as in treatment of TB infections of the central nervous system. Novel drug design by substituent substitution has produced highly successful cephalosporin antibiotics ${ }^{25}$ and effective antiviral drugs that are analogues to acyclovir. ${ }^{26}$ Previous studies have concluded that the substitution of carefully selected structure substituents can favorably modify water solubility, polar surface area, molecular weight, $\log \mathrm{P}$, and the metabolic fate of drugs. ${ }^{27}$ Considering the apparent threat posed by drug resistant $\mathrm{TB}$, the utilization of data mining to enhance the discovery of novel drug design appears efficacious, and should produce viable new drug candidates for consideration in the clinical treatment of Mycobacterium tuberculosis. In summation, seventeen novel hydrazide compounds were identified following similarity-substituent data mining utilizing Molinspiration algorithmic analysis. Vital pharmaceutical properties are elucidated to ascertain underlying relationships by pattern recognition methodology to discern potential clinical employment. Utilizing isoniazid as the parent structure the constructs developed demonstrated variation in properties necessary for favorable druglikeness and bioavailability. Antiviral and antibacterial drug design has highly benefitted through consideration of substituent substitution formulation. The PSA values of the agents discussed here vary from $55.121 \mathrm{~A}^{2}$ to $94.036 \mathrm{~A}^{2}$, indicative of an expected intestinal absorption that varies from $>85 \%$ to approximately $35 \%$, respectively. A number of analogs show potential for blood-brain barrier penetration and targeting of TB infections located within the central nervous system, this feature due to a polar surface area of $60 \mathrm{~A}^{2}$ to $70 \mathrm{~A}^{2}$. This criteria is met by agents 1 (isoniazid), 2, 3, 5, 6, 7, 8, and 17; and therefore useful for the treatment of Mycobacterium tuberculosis infections of the central nervous system. An ANOSIM result of $R=0.2315$, indicates an appreciable level of similarity among these compounds. However, discriminant function analysis elucidated two groups within this population of anti-tuberculosis agents as follows: 1) Agent 1 (isoniazid), 3, 4, 7, 8, 11, 12 , 13, 14, and 17; 2) Agent 2, 5, 6, 9, 10, 15, 16, and 18. Data mining techniques have advance substantially and are able to produce viable new drug candidates for consideration for the clinical treatment of TB. ${ }^{7,23,27}$ Seventeen hydrazide compounds (success rate of approximately $38 \%$ ) having diverse molecular properties resulted from substituent data mining with potential for clinical application.

\section{References}

1. Kumar V, Vinay AK, Fausto N, Mitchell RN. Robbins basic pathology. 8th ed. New York: Sauders Elsevier; 2007.

2. Konstantinos A. Testing for tuberculosis. Australian Presecriber 2010;33:12-8.

3. Jasmer RM, Nahid P, Hopewell PC. Clinical practice. Latent tuberculosis infection. $\mathrm{N}$ Engl J Med 2002;72:213-20.

4. Cox R. Quantitative relationships for specific growth rates and macromolecular compositons of Mycobacterium tuberculosis, Streptomyces coelicolor A3(2) and Escherichia coli B/r: an integrative theoretical approach. Microbiol 2004;150:141326 .

5. Madison B. Application of stains in clinical microbiology. Biotech Histochem 2001;76: 119-25.

6. Soolingen DV, Hoogenboezem T, De Haas $P$ et al. A novel pathogenic taxon of the Mycobacterium tuberculosis complex, Canetti; characterization of an exceptional isolate from Africa. Int $\mathbf{J}$ of Syst Bacteriol 1997;47:1236-45.

7. Tomioka H. Current status of some antituberculosis drugs and the development of new antituberculous agents with special reference to their in vitro and in vivo antimicrobial activites. Curr Pharm Des 2006;12:4047-70.

8. Chiang CY, Centis R, Migliori G. Drugresistant tuberculosis: past, present, and future. Respirology 2010;15:413-32.

9. Da Silva Almeida P, Palomino JC. Molecular basis and mechanisms of drug resistance in Mycobacterium tuberculosis: classical and new drugs. J Antimicrob Chemother 2011;66:1417-30.

10. Lawson L, Yassin MA, Abdurrahman ST et al. Resistance to first-line tuberculosis drugs in three cities of Nigeria. Trop Med Int Health 2011 [Epub ahead of print]

11. Kozhamkulov U, Akhetova A, Rakhimova S et al. Molecular characterization of rifampicin-and isoniazid-resistant Mycobacterium tuberculosis strains isolated in Kazakhastin. J Infect Dis 2011;64: 253-55.

12. Alcaide F, Santin M. Multidrug-resistant tuberculosis. Enferm Infect Microbiol Clin 2008;26:54-60.

13. Sharma SK, George N, Kadhiravan T et al. Prevalence of extensively drug-resistant tuberculosis among patients with multidrug-resistant tuberculosis: a retrospective hospital-based study. Indian J Med Res 2009;130:392-95.

14. Shah NS, Pratt R, Armstrong L et al. Extensively drug-resistant tuberculosis in the United States, 1993-2007. JAMA $2008 ; 300 ; 2153-60$. 
15. Banerjee R, Schecter GF, Flood J, Porco TC. Extensively drug-resistant tuberculosis: new strains, new challenges. Expert Rev Anti-Infect Ther 2008;6:713-24.

16. Jain A, Mondal R. Extensively drug-resistant tuberculosis: current challenges and threats. FEMS Immunol Med Microbiol 2008;53:145-50.

17. Agrawal V, Patgaonkar PR, Nagariva SP. Tuberculosis of spine. J Craniovertebr Junction Spine 2010;1:74-85.

18. Pawar UM, Kundnani V, Agashe V et al. Multidrug-resistant tuberculosis of the spine-is it the beginning of the end? A study of twenty-five culture proven multidrug-resistant tuberculosis spine patients. Spine 2009;34:806-10.

19. Bartzatt R. Properties and potency of small molecule agents for treatment of Mycobacterium tuberculosis infections of the central nervous system. Cent Nerv Syst Agents Med Chem 2011;11:66-72.

20. Ertl P, Rohde B, Selzer P. Fast calculation of molecular polar surface area as a sum of fragment-based contributions and its application to the prediction of drug transport properties. J Med Chem 2000;43:37147.

21. Lipinski CA, Lombardo F, Dominy BW, Feeney PJ. Experimental and computational approaches to estimate solubility and permeability in drug discovery and development settings. Adv Drug Del Rev 2001;46:3-26.

22. Kelder J, Grootenhuis P, Bavada D et al. Polar molecular surface as a dominating determinant for oral absorption and brain penetration of drugs. Pharm Res 1999;16: 1514-9.

23. Hansch C, Unger SH. Strategy in drug design. cluster analysis as an aid in the selection of substituents. J Med Chem 1973;16:1217-22.

24. Clarke KR. Non-parametric multivariate analysis of changes in community structure. Australian J of Ecology. 1993;18:117143.

25. Price KE, McGregor DN. Basic design of beta-lactam antibiotics-cephalosporins. Scan J Infect Dis 1984;42:50-63.

26. Golankiewicz B, Ostrowski T, Andrei G et al. Tricyclic analogues of acyclovir and ganciclovir. Influence of substituents in the heterocyclic moiety on the antiviral activity. J Med Chem 1994;37:3187-90.

27. Hajduk PJ, Greer J. A decade of fragmentbased drug design: strategic advances and lessons learned. Nature Rev Drug Discov 2007;6:211-9. 